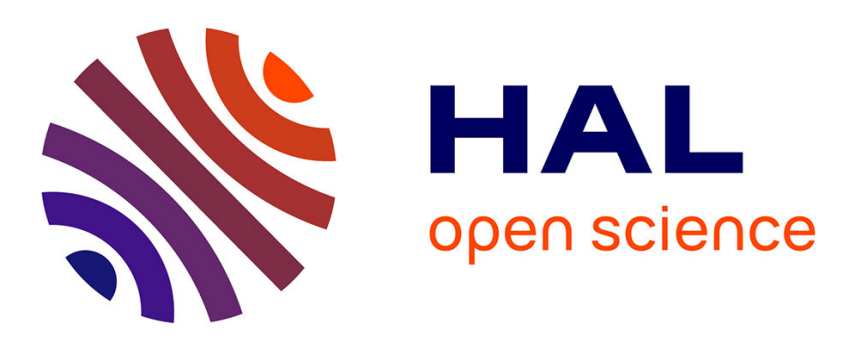

\title{
Joint Probability of Shape and Image Similarities to Retrieve 2D TRUS-MR Slice Correspondence for Prostate Biopsy
}

Jhimli Mitra, Soumya Ghose, Désiré Sidibé, Robert Marti, Arnau Oliver, Xavier Llado, Joan C. Vilanova, Josep Comet, Fabrice Mériaudeau

\section{To cite this version:}

Jhimli Mitra, Soumya Ghose, Désiré Sidibé, Robert Marti, Arnau Oliver, et al.. Joint Probability of Shape and Image Similarities to Retrieve 2D TRUS-MR Slice Correspondence for Prostate Biopsy. IEEE Conference of the Engineering in Medicine and Biology Society, Aug 2012, San Diego, United States. hal-00710941

\section{HAL Id: hal-00710941 https://hal.science/hal-00710941}

Submitted on 22 Jun 2012

HAL is a multi-disciplinary open access archive for the deposit and dissemination of scientific research documents, whether they are published or not. The documents may come from teaching and research institutions in France or abroad, or from public or private research centers.
L'archive ouverte pluridisciplinaire HAL, est destinée au dépôt et à la diffusion de documents scientifiques de niveau recherche, publiés ou non, émanant des établissements d'enseignement et de recherche français ou étrangers, des laboratoires publics ou privés. 


\title{
Joint Probability of Shape and Image Similarities to Retrieve 2D TRUS-MR Slice Correspondence for Prostate Biopsy
}

\author{
J. Mitra ${ }^{1,2}$, S. Ghose ${ }^{1,2}$, D. Sidibé ${ }^{1}$, R. Martî ${ }^{2}$, A. Oliver ${ }^{2}$, X. Lladó $^{2}$, \\ J. C. Vilanova ${ }^{3}$, J. Comet ${ }^{4}$ and F. Meriaudeau ${ }^{1}$
}

\begin{abstract}
This paper presents a novel method to identify the 2D axial Magnetic Resonance (MR) slice from a pre-acquired MR prostate volume that closely corresponds to the $2 \mathrm{D}$ axial Transrectal Ultrasound (TRUS) slice obtained during prostate biopsy. The method combines both shape and image intensity information. The segmented prostate contours in both the imaging modalities are described by shape-context representations and matched using the Chi-square distance. Normalized mutual information and correlation coefficient between the TRUS and MR slices are computed to find image similarities. Finally, the joint probability values comprising shape and image similarities are used in a rule-based framework to provide the MR slice that closely resembles the TRUS slice acquired during the biopsy procedure. The method is evaluated for 20 patient datasets, of which 18 results match at least one of the two clinical expert choices.
\end{abstract}

\section{INTRODUCTION}

Prostate cancer has been a major cause of mortality among human males in the European and American societies since the last 25 years. Therefore, prostate cancer screening programs are conducted where a patient with abnormal findings after a digital rectal examination, serum Prostate Specific Antigen (PSA) level over 4.0ng/ml and PSA velocity between $0.4-0.75 \mathrm{ng} / \mathrm{ml} / \mathrm{yr}$ is generally advised for a prostate biopsy for histopathological examination of the prostate tissues. The appearance of malignant lesions in a Transrectal Ultrasound (TRUS) guided needle biopsy is mostly hypoechoic and the accuracy of finding such lesions is typically $43 \%$ in sonography [1]. Approximately $25 \%-42 \%$ of cancer lesions can also be isoechoic under TRUS. Therefore, the chance to diagnose hypoechoic lesions from TRUS guided biopsy that are malignant is $\leq 57 \%$ [2]. Vilanova et al. [3] demonstrated that the accuracy of Magnetic Resonance Imaging (MRI) to diagnose prostate cancer is $72 \%-76 \%$. Therefore, MRI may serve as a triage for men deemed to be at risk of prostate cancer and fusion of pre-biopsy MR images onto interventional TRUS images

This work was supported by VALTEC 08-1-0039 of Generalitat de Catalunya, Spanish Science and Innovation grant nb. TIN2011-23704, Spain and Conseil Régional de Bourgogne, France.

1 J. Mitra, S. Ghose, D. Sidibé \& F. Meriaudeau are with Le2i CNRS-UMR 6306, Université de Bourgogne, Le Creusot, France. jhimli.mitra@u-bourgogne.fr, soumya.ghose@u-bourgogne.fr, drodesire.sidibe@u-bourgogne.fr,fabrice.meriaudeau@u-bourgogne.fr

2 R. Martí, A. Oliver \& X. Lladó are with the Computer Vision and Robotics Group, University of Girona, Girona, Spain.marly@eia.udg.edu, aoliver@eia.udg.edu, llado@eia.udg.edu

3 J. C. Vilanova is with the Girona Magnetic Resonance Center, Girona, Spain.

${ }^{4}$ J. Comet is with the Hospital Dr. Josep Trueta, Girona, Spain. might increase the overall biopsy accuracy [4].

Fusion of pre-biopsy MR on interventional TRUS may be done in several ways. An Electro Magnetic (EM) tracker attached to the 2D TRUS probe may be used that sweeps the prostate to reconstruct a 3D TRUS volume. The 3D TRUS volume is then fused with the MR volume to obtain the spatial position of the 2D TRUS slice during biopsy within the pre-biopsy MR volume [4]. On the other hand, a 3D TRUS probe may be directly used to acquire 3D TRUS volume and a volume-volume registration may be performed [5]. However, neither 3D TRUS probe is commonly available in diagnostic centers nor the use of the EM tracker is an established clinical practice. Therefore, intending to solve the 2D TRUS-MR slice correspondence problem, we propose a method based on Chi-square distance of shape-context representations of the prostate contours and image similarity measures like Normalized Mutual Information (NMI) and Correlation Coefficient (CC) of the TRUS-MR slices. The probability of an MR slice being the correct match for the corresponding TRUS slice is determined from the joint probabilities of shape similarity and each of the image similarity measures (NMI and CC) yielding two sets of probability values. The shape and image similarities assume independence, therefore multiplication of the same provides the combined probability. The slice having the maximum joint probability among the obtained sets of probability values is normally chosen as the correct match. However, owing to the segmentation inaccuracies and inter-modality prostate deformations, the overlap area between the TRUS and MR images will differ that would incorporate some error in the image-based similarity and hence the choice of the correct MR slice from joint shape and image similarities. Therefore, a rule-based approach is adopted to prioritize the shape similarity in such cases over image similarities. The novelties of the proposed work may be summarized as follows:

1) Using shape context representations of the contours to find prostate shape similarities between TRUS and MR slices.

2) Combining shape information (here shape context) with image intensity information (NMI and CC), thereby yielding the combined probability of an MR slice that closely resembles the TRUS slice both in shape and intensity pattern.

3) Rule-based approach to prioritize the shape similarity in case of ambiguous maximum joint probability values of shape and image similarities.

In the remaining paper, section II describes the proposed 


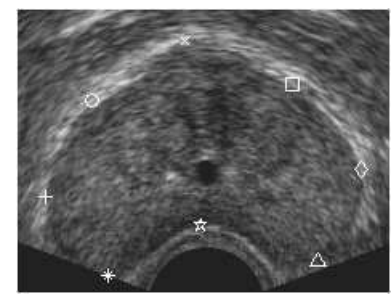

(a)



(b)
Fig. 1. Point correspondences example. (a) contour points in TRUS, (b) point correspondences of (a) in MR

method in detail, section III provides the results and discussions followed by the conclusions in section IV.

\section{THE METHOD}

In this work, the prostate is manually segmented from the 2D TRUS axial slice and the pre-biopsy axial MR volume where the TRUS slices are considered to be parallel to the MR axial plane. The manual segmentation ensures better evaluation of our method, although in future we will use the fast automatic prostate segmentation methods in both MR and US modalities by Ghose et al. [6], [7]. The shape similarity measure using Chi-square distance is explained in section II-A, the image similarity measures like NMI and $\mathrm{CC}$ are explained in section II-B with the explanation of joint probability of shape and image similarities and the rule-based approach to choose the best matching MR slice corresponding to the axial TRUS slice in section II-C.

\section{A. Shape Similarity}

The segmented prostate contour points are uniformly sampled using fixed Euclidean distance of $\varepsilon$ i.e. if $c_{i}$ is a contour point, $i=1, \ldots, N$, then maximize the following equation

$$
\underset{j}{\arg \max }\left\|c_{i}-c_{j}\right\|^{2} \leq \varepsilon, \quad i \neq j .
$$

Let the number of uniformly sampled points now be represented as $n$, then each sample point $c_{i}$ may be represented by a shape descriptor that is a $n-1$ length vector of logpolar relative distances to points $c_{j}$, where $i \neq j$. The shape descriptor is binned into a histogram that is uniform in log-polar space and this histogram is the shape-context representation of a contour point [8] i.e. $c_{i}$ is represented by a histogram $h_{i}(k, \theta)$ such that

$$
h_{i}(k, \theta)=\#\left\{c_{j}, i \neq j \quad: \quad\left(c_{i}-c_{j}\right) \in \operatorname{bin}(k, \theta)\right\} .
$$

$k$ is the $\log r=\log \left(\sqrt{\left(x_{i 1}-x_{j 1}\right)^{2}+\left(x_{i 2}-x_{j 2}\right)^{2}}\right)$ and $\theta=\tan ^{-1} \frac{x_{j 2}-x_{i 2}}{x_{j 1}-x_{i 1}}$ of the relative distance $\left(c_{i}-c_{j}\right)$, where, $c_{i}=\left(x_{i 1}, x_{i 2}\right)$ and $c_{j}=\left(x_{j 1}, x_{j 2}\right)$. As suggested by Belongie et al. [8], a total of 5 bins are considered for $k$ and 12 bins for $\theta$ that ensures that the histogram is uniform in log-polar space. This also means that more emphasis is given to the nearby sample points than those that are far away.

In the original work of Belongie et al. [8], the point correspondence between two shapes is obtained by a bipartite graph matching method. However, in this work we choose the
Bhattacharyya distance [9] between the shape-context histograms of two shapes to find the best point correspondence since it is fast to compute and statistically a robust measure to find correspondences in similar shapes like prostate contours in TRUS and MRI. Thus, to match a point $c_{i}$ in a shape to a point $c_{j}^{\prime}$ in another shape, the Bhattacharyya coefficients between the shape-context histogram of $c_{i}$ and all $c_{j}^{\prime}$ are computed and the $c_{j}^{\prime}$ that maximizes the relation in Eq. (3) is chosen as the corresponding point.

$$
\underset{c_{j}^{\prime}}{\arg \max } \sum_{k=1}^{5} \sum_{\theta=1}^{12} \sqrt{\hat{h}_{i}(k, \theta) \cdot \hat{h}_{j}^{\prime}(k, \theta)},
$$

where, $\hat{h}_{i}(k, \theta)$ and $\hat{h}_{j}^{\prime}(k, \theta)$ are the normalized shapecontext histograms of $c_{i}$ and $c_{j}^{\prime}$ respectively. Fig. 1 shows the contour correspondences overlaid on the TRUS and MR prostate shapes.

After the corresponding points are identified, the Chisquare $\left(\chi^{2}\right)$ distances between the TRUS slice and each of the MR slices are calculated based on the corresponding shape-context histograms and is given by $\mathcal{C}_{i j}$ in Eq. (4). The final distance is the sum of all the $\chi^{2}$ distances of the corresponding points (shape-context histograms) in TRUS and $\mathrm{MR}$ and is given by $\mathcal{H}$ in the following equation.

$$
\mathcal{C}_{i j}=\frac{1}{2} \sum_{k=1}^{5} \sum_{\theta=1}^{12} \frac{\left(\hat{h}_{i}(k, \theta)-\hat{h}_{j}^{\prime}(k, \theta)\right)^{2}}{\hat{h}_{i}(k, \theta)+\hat{h}_{j}^{\prime}(k, \theta)}, \quad \mathcal{H}=\sum_{i=1}^{l} \mathcal{C}_{i j},
$$

where, $l$ is the number of point correspondences. The TRUSMR slice pair with minimum sum of $\chi^{2}$ distance $(\mathcal{H})$ is identified and its significance will be discussed in the following subsection.

\section{B. Image Similarities}

Image similarity measures have been extensively used in multimodal image registration problem to ensure that the moving image is transformed with close resemblance to the fixed image. In this work, our problem is to find an MR slice in the volume that closely resembles the TRUS slice. Therefore, to find such similarity we employ the wellknown NMI and CC as image similarity measures. Fei et al. [10] demonstrated that $\mathrm{CC}$ is more discriminative as an image similarity in low resolutions and NMI at higher resolutions for registration problems. Related to our problem, some TRUS slices have smaller prostate sizes than the other. Therefore, considering the variability in prostate sizes we decided to use both NMI and CC as image similarity measures.

The TRUS-MR slice pair identified with the minimum $\mathcal{H}$ as obtained from Eq. (4) is used to retrieve the 2D rigid transformation (in-plane rotation and translation) parameters between them; and the remaining MR slices in the volume are rigidly registered with the TRUS slice using the same transformation parameters. This registration step ensures similar 2D in-plane rigid alignment of all the MR slices of the volume with the 2D TRUS slice. After the alignment of the MR volume with the TRUS slice, pairwise NMI and $\mathrm{CC}$ are computed for each MR-TRUS slice pair. The NMI is 
TABLE I

EXPERT CHOICES AND THE RESULTS FOR THE MR SLICES CORRESPONDING TO A TRUS SLICE OBTAINED BY OUR METHOD. THE MATCHED CASES

ARE SHOWN IN ITALICS.

\begin{tabular}{|c|c|c|c|c|c|c|c|c|c|c|c|c|c|c|c|c|c|c|c|c|c|}
\hline Patients/MR Slice & 1 & 2 & 3 & 4 & 5 & 6 & 7 & 8 & 9 & 10 & 11 & 12 & 13 & 14 & 15 & 16 & 17 & 18 & 19 & 20 & Agreement (\%) \\
\hline Expert 1 & 6 & 8 & 9 & 7 & 6 & 10 & 6 & 10 & 5 & 7 & 6 & 5 & 12 & 8 & 6 & 5 & 7 & 7 & 6 & 7 & $\mathbf{6 5 \%}$ \\
\hline Expert 2 & 2 & 7 & 6 & 5 & 6 & 9 & 6 & 8 & 7 & 6 & 6 & 4 & 13 & 8 & 4 & 8 & 10 & 9 & 6 & 7 & $\mathbf{8 0 \%}$ \\
\hline Our method & 3 & 8 & 3 & 6 & 5 & 9 & 6 & 8 & 7 & 6 & 6 & 5 & 13 & 8 & 6 & 8 & 10 & 6 & 6 & 4 & - \\
\hline
\end{tabular}

an information theoretic measure that measures the mutual dependence between the images [11] and is given by

$$
\mathrm{NMI}=\frac{H(M)+H(T)}{H(M, T)}
$$

where, $H(M)$ and $H(T)$ are the marginal entropies of the MR $(M)$ and TRUS $(T)$ images respectively, and $H(M, T)$ is the joint entropy of the images. $H(M, T)$ can be written using probability theory as

$$
H(M, T)=-\sum_{M, T} p(M, T) \log [p(M, T)],
$$

where, $p(M, T)$ is the joint probability distribution of the images obtained from their joint histogram.

The CC gives a linear dependence between two random variables $M(m)$ and $T(t)$ [12] as intensities of the MR and the TRUS images respectively, and is defined as:

$$
C C(M, T)=\frac{\sum(T(t)-\bar{T}(t))(M(m)-\bar{M}(m))}{\sqrt{\sum(T(t)-\bar{T}(t))^{2} \sum(M(m)-\bar{M}(m))^{2}}} .
$$

$m$ and $t$ are the pixel positions in the TRUS and MR images respectively. $\bar{M}(m)$ and $\bar{T}(t)$ are the average pixel intensities for the overlapping regions.

\section{Choosing the best matching MR slice}

The MR slice corresponding to the observed TRUS slice should ideally be the one with lowest $\mathcal{H}$ obtained from section II-A and with maximized NMI or CC as obtained from section II-B. The values of these statistical shape and image similarity measures are consecutively transformed into pdfs to compute the joint probability.

Given a set of independent random variables $\mathbf{X}=$ $\left\{\mathbf{x}_{1} \ldots \mathbf{x}_{n}\right\}$, each defined by the pdf $p\left(\mathbf{x}_{i}\right), i=1 \ldots n$, the joint probability of the independent random variables is given by

$$
p\left(\mathbf{X}=\mathbf{x}_{1}, \ldots \mathbf{X}=\mathbf{x}_{n}\right)=p\left(\mathbf{X}=\mathbf{x}_{1}\right) \cdot \ldots \cdot p\left(\mathbf{X}=\mathbf{x}_{n}\right)
$$

In this work, the set of independent random variables is $\mathbf{X}=$ $\left\{\mathcal{H}^{\prime}, \mathrm{NMI}, \mathrm{CC}\right\}$, where $\mathcal{H}^{\prime}=1-\mathcal{H}$ and their respective probability values constituting the pdfs. Therefore, two sets of joint probability values are obtained by combining the shape and image similarities as

$$
P_{\mathrm{NMI}}=p\left(\mathbf{X}=\mathcal{H}^{\prime}, \mathbf{X}=\mathrm{NMI}\right)=p\left(\mathbf{X}=\mathcal{H}^{\prime}\right) \cdot p(\mathbf{X}=\mathrm{NMI})
$$

$$
P_{\mathrm{CC}}=p\left(\mathbf{X}=\mathcal{H}^{\prime}, \mathbf{X}=\mathbf{C C}\right)=p\left(\mathbf{X}=\mathcal{H}^{\prime}\right) \cdot p(\mathbf{X}=\mathbf{C C})
$$

After obtaining the joint probabilities, the idea is to find the MR slice that corresponds to the TRUS slice jointly maximizing the $\mathcal{H}^{\prime}$ and NMI or $\mathcal{H}^{\prime}$ and CC. Let us consider the maximum joint probabilities of $P_{\mathrm{NMI}}$ and $P_{\mathrm{CC}}$ be
$P_{\mathrm{NMI}}^{\max }$ and $P_{\mathrm{CC}}^{\max }$ respectively. Then the rule-based method to identify the best MR slice is as follows: The value of $\lambda$ is

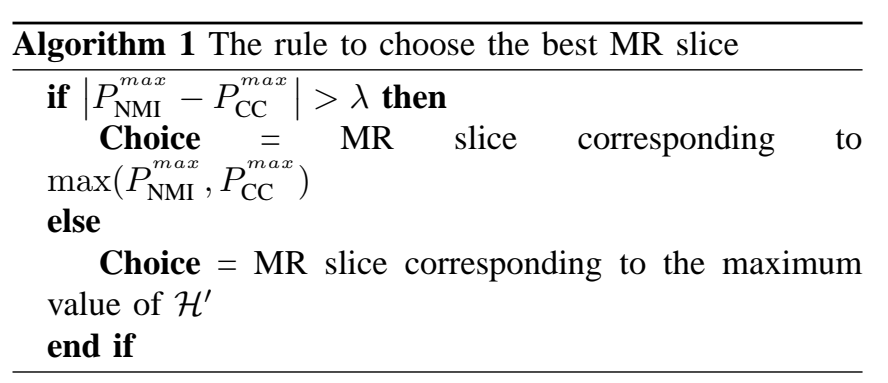

determined through the experimental validation procedure. If $P_{\mathrm{NMI}}^{\max }$ and $P_{\mathrm{CC}}^{\max }$ both have closely separated values then it is difficult to bias on any one of the joint probability $\left(P_{\mathrm{NMI}}^{\max }\right.$ or $\left.P_{\mathrm{CC}}^{\max }\right)$ to determine the best matching MR slice. Therefore, in such cases the shape similarity measure is prioritized in determining the slice choice.

\section{RESULTS AND DISCUSSIONS}

The results are validated against the MR slice choices obtained from an expert radiologist and an expert urologist for 20 patients axial mid-gland TRUS slices. The axial MR slices have slice thickness of $3 \mathrm{~mm}$ with inter-slice gap of $3.5 \mathrm{~mm}$ where the pixel dimension is $0.2734 \mathrm{~mm} \times 0.2734$ $\mathrm{mm}$. The value of $\lambda$ is determined experimentally as 0.15 that is the value which maximizes the number of cases in agreement to that of the expert choices. This means that if the maximum joint probability values $P_{\mathrm{NMI}}^{\text {max }}$ and $P_{\mathrm{CC}}^{\text {max }}$ are similar by more than $85 \%$ then the slice choice is dependent only on the maximum shape similarity rather than joint image and shape similarities. Table I shows the choices of the axial MR slice corresponding to an axial TRUS slice provided by the experts (independently) and the results we obtained using our method. The inter-slice gap being $3.5 \mathrm{~mm}$, we have considered the $[-1,+1]$ slices i.e. a statistically significant $20 \%$ error when computing the inter-expert and between expert and our method accuracies of slice choice.

It is observed from Table I that the automatic MR slice choice matched at least one of the expert choices for 18 cases wherein the experts agree in their choices for 11 patient cases (patients 2,5,6,7,10,11,12,13,14,19 \& 20). The expert choices matched exactly in 6 cases $(5,7,11,14,19$ \& 20) out of which our result matched exactly for 4 patient cases $(7,11,14 \& 19)$ and -1 slice away for patient 5 respectively. Since, the expert choices agreed exactly and $[-1,+1]$ slice away in 11 out of 20 cases, they are in agreement of $55 \%$.

Comparing each of the expert choices independently 

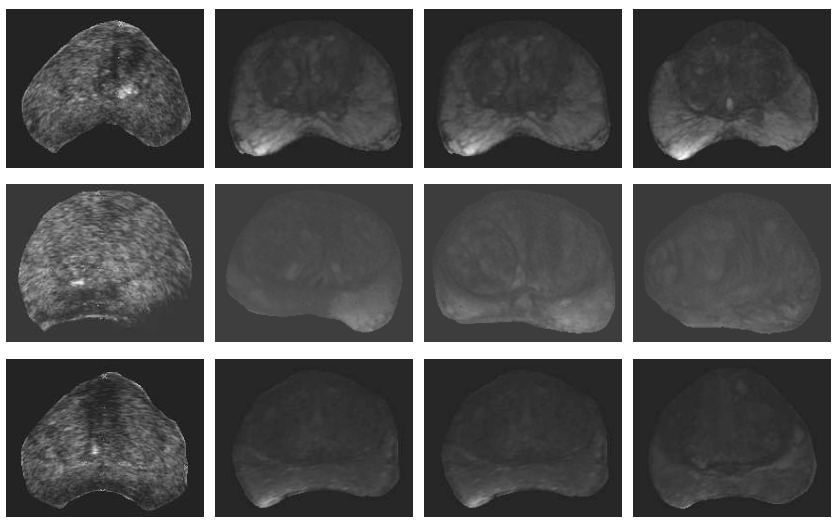

Fig. 2. TRUS-MR corresponding slices. Rows (top to bottom) show patient cases 5,3 and 20 respectively. The $1^{\text {st }}$ column shows the TRUS slices, the $2^{\text {nd }}$ and the $3^{\text {rd }}$ show the expert 1 and 2 MR slice choices respectively and the last column shows the obtained result using our method.

with our method, the exact matches with expert 1 are for 7 patients $(2,7,11,12,14,15 \& 19)$ while $[-1,+1]$ slice away for 6 patients $(4,5,6,10,13$, \& 18). Therefore, 13 out of 20 cases i.e. $65 \%$ results are in agreement with that of the choices of expert 1. Similarly for 11 cases $(6,7,8,9,10,11,13,14,16,17 \& 19)$ our results exactly matched expert 2 choices and are $[-1,+1]$ slice away for 5 patient cases $(1,2,4,5, \& 12)$. This signifies that the results of our method are in $80 \%$ agreement with that of expert 2 slice choices. The agreements between our method and each of the expert choices are shown in the last column of Table I. The inter-expert variability in the choice of MR slice being high ( $55 \%$ agreement), our method performs better with an agreement of $65 \%$ for expert 1 that shows a $18.18 \%$ increase in performance and with $80 \%$ agreement for expert 2 that shows an increase in performance by $45 \%$. The overall performance of our method is said to be $90 \%$ considering accurate slice matches in 18 out of 20 cases while failing for patient 3 and 20. Fig. 2 shows patient case 5 where the result obtained is one slice below than that of the choices of experts 1 and 2 as shown in Table I. Fig. 2 also shows patient cases 3 and 20 where the results are not close to any of the expert choices. However, observing the slice choice by our method and that of the expert for patient 20 it may be noted that both the choices are visually similar.

The method has been implemented in MATLAB and the complete process takes 3 secs on an average to find out the corresponding MR slice from a set of $12-14$ slices. It is to be noted that Xu et al. [4] employed an EM tracker to locate the spatial position of the 2D TRUS slice (during biopsy) in the 3D TRUS volume. Thereafter, to compensate for the prostate motion, the sum-of-squared differences (SSD) between the maximum translational and rotational TRUS slices within a short time frame of the biopsy and the corresponding spatial 2D TRUS slices obtained in the 3D TRUS volume was minimized. Similarly, considering an error of $[-1,+1]$ slices from the actual MR slice, we can directly use the slice obtained by our proposed method for multimodal registration between TRUS and MR employing the method of Mitra et al. [13]. However, if an EM tracker is additionally attached to the TRUS probe during biopsy, then it would be possible to locate an approximate position of the TRUS slice in MR volume. Consequently, our method can be employed to search for the best slice within a smaller subset in the neighborhood of the correct MR slice thereby improving on the accuracy of slice choice.

\section{CONCLUSIONS}

A method to find out 2D MR slice correspondence of a 2D axial TRUS slice during biopsy has been reported in this paper. The method is based on statistical shape and image similarity measures and their joint probabilities and applying a rule-based method to prioritize the shape similarity in some cases. The method is fast in finding out MR correspondences that are nearly the same as the choices obtained from two experts. Since EM tracker is not easily available in hospitals in Europe and 3D TRUS is normally not employed for biopsy purposes, our proposed method may provide a good starting point for multimodal fusion of TRUS-MR images to improve the sampling of biopsy tissues. Although the results reported in this paper are validated only for mid-gland or close to midgland axial slices, the validations for the base/apex TRUS axial slices, TRUS sagittal slices and cross-validation of our method with the use of an EM tracker have been left as future works.

\section{REFERENCES}

[1] P. Carroll and K. Shinohara, "Transrectal ultrasound guided prostate biopsy," Tech. Rep., Dept. of Urology, Univ. of California, San Francisco, 2010, http://urology.ucsf.edu/patientGuides.html, accessed [30th Dec, 2010].

[2] H. A. Bogers et al., "Contrast-enhanced three-dimensional power Doppler angiography of the human prostate: correlation with biopsy outcome," Urology, vol. 54, no. 1.

[3] J. C. Vilanova et al., "Usefulness of prebiopsy multi-functional and morphologic MRI combined with the free-to-total PSA ratio in the detection of prostate cancer," Am. Jour. of Roentgen., vol. 196, no. 6, pp. W715-W722, 2011.

[4] S. Xu et al., "Real-time MRI-TRUS fusion for guidance of targeted prostate biopsies," Comp. Aid. Surg., vol. 13, no. 5, pp. 255-264, 2008.

[5] M. Baumann et al., "Prostate biopsy tracking with deformation estimation," Med. Imag. Anal., In Press, 2011.

[6] S. Ghose et al., "A hybrid framework of multiple active appearance models and global registration for 3D prostate segmentation in MRI," in Proc. SPIE Med. Imag., February 2012, vol. 8314, pp. 8314S-1-9.

[7] S. Ghose et al., "Statistical shape and texture model of quadrature phase information for prostate segmentation," in IJCARS, vol. 7, pp. 43-55, 2012.

[8] S. Belongie et al., "Shape matching and object recognition using shape contexts," IEEE Trans. on Patt. Anal. and Mach. Intell., vol. 24, no. 4, pp. 509-522, 2002.

[9] A. Bhattacharyya, "On a measure of divergence between two statistical populations defined by their probability distribution," Bull. of the Calcutta Math. Soc., vol. 35, pp. 99-110, 1943.

[10] B. W. Fei et al., "Slice-to-volume registration and its potential application to interventional MRI-guided radio-frequency thermal ablation of prostate cancer," IEEE Trans. on Med. Imag., vol. 22, no. 4, pp. 515-525, 2003.

[11] C. Studholme et al., "An overlap invariant entropy measure of 3D medical image alignment," Patt. Recog., vol. 72, no. 1, pp. 71-86, 1999.

[12] W. H. Press et al., Numerical recipes in C: The Art of Scientific Computing, Cambridge Univ. Press, London, UK, 2nd edition, 1993.

[13] J. Mitra et al., "A Non-linear Diffeomorphic Framework for Prostate Multimodal Registration," in Proc. of DICTA, December 2011, pp. 31-36. 